\title{
Some Experiments in Supervised Pattern Recognition with Incomplete Training Samples
}

\author{
Ricardo Barandela ${ }^{1}$, Francesc J. Ferri ${ }^{2}$, and Tania Nájera ${ }^{1}$ \\ ${ }^{1}$ Lab for Pattern Recognition, Instituto Tecnológico de Toluca, México \\ C.P. 52140, Metepec, Estado de México \\ rbarandela@hotmail.com \\ ${ }^{2}$ Dept. d'Informàtica, Universitat de València \\ 46100 Burjassot, València, Spain
}

\begin{abstract}
This paper presents some ideas about automatic procedures to implement a system with the capability of detecting patterns arising from classes not represented in the training sample. The procedure aims at incorporating automatically to the training sample the necessary information about the new class for correctly recognizing patterns from this class in future classification tasks. The Nearest Neighbor rule is employed as the central classifier and several techniques are added to cope with the peril of incorporating noisy data to the training sample. Experimental results with real data confirm the benefits of the proposed procedure.
\end{abstract}

\section{Introduction}

Supervised pattern recognition methods are based on the information supplied by a training sample (TS). It is usually assumed that this TS satisfies two important conditions:

1. Each member is a pattern that has been identified as representative of one of the classes and is actually a member of that class.

2. The $c$ classes represented in the training sample span the entire set of the classes that need to be considered in the problem at hand.

Experience in practical applications has shown that these assumptions do not always hold and that violation of one or both of them may seriously degrade classification accuracy of the recognition system. Particularly sensitive to these deficiencies are nonparametric classifiers whose learning is not based upon any assumption about probability density functions.

In several real domains, class identification of the training patterns is a difficult and very costly task. Also, the fact that one (or some) of the classes is not known a priori leads to situations lying between supervised and unsupervised methods that have already been coined as partially exposed environments [2]. 
In this paper, the situation in which one of the classes is missing from the original TS is taken into account. This situation is common in some fields as Remote Sensing in which data from one zone is used to classify other zones (or the same zone but at a different time) in which new classes may appear. Another similar situation arises in Banknote classification when trying to detect forgery. Although patterns from missing classes can always be treated as outliers (with regard to the available information) it is quite clear that this is not the best option in real situations where a classification system is continuously running and receiving new data that has potentially the ability of giving us information about possible new classes.

The problem statement is challenging in itself and has a straightforward real application. In this paper, we try to contribute with a preliminary solution based on prototype selection techniques for the nearest neighbor rule. To this end, we must first detect the situation in which a running system misclassifies patterns that may belong in fact to a class about which no information is available. Second, we must put the information collected in this first classification stage in such a way that the system can update the TS to include particular samples belonging to the new class. Both of this are challenging and difficult problems to be solved in general. On the other hand, there are several different ways for dealing with these situations. In this paper, some of these will be used and put together to deal with a simplified version of the problem.

The paper is organized as follows. In section 2, basic ideas about NN rules are introduced. Several proposals to the problem of extracting information about missing classes are introduced in sections 3 and 4. Section 5 explains the experiments carried out and the paper ends with the conclusions in section 6 .

\section{The NN Rule and Some Related Techniques}

The Nearest Neighbor (NN) rule is one of the oldest and better-known algorithms for nonparametric classification. The entire TS is stored in the computer memory. To classify a new instance, its distance is computed to each one of the stored training cases. The new instance is then assigned to the class represented by its nearest neighboring training pattern. The NN rule is very popular because of: a) conceptual simplicity, b) easy implementation, c) known error rate bounds and d) potentiality to compete favorably with other classification methods in real data applications.

The performance of the NN rule can be improved in practice by using prototype selection techniques. Wilson [3] proposed a procedure (Editing) that consists of applying the $k$-NN classifier to estimate the class label of all prototypes in the TS and discard those instances whose class label does not agree with the class associated with the largest number of the $k$ neighbors. Most prototype selection techniques share the general idea of discarding prototypes that significantly deviate from the general tendency in their class and also prototypes lying in the overlapping zones between classes. In this way, the processed TS is able to classify new patterns in a close-tooptimal way. Apart from its optimal behavior, editing prototypes should be almost compulsory in situations in which an optimal (or at least fair) labeling of the training is impossible because it relies in a very difficult or costly (usually manual) task. These situations have been previously referred to as imperfectly supervised [1]. Many 
theoretical and empirical results have corroborated the convenience of selecting training patterns in this way [4-7].

The idea of discarding can be extended to consider changing the class labels apart from removing patterns (Generalized Edition, [9]). In this way, the obtained TS may still perform well (or at least better) in the small sample size situation, which is one of the drawbacks of editing in practice. In short, Generalized Edition looks for modifications of the training sample structure through changes of the labels (reidentification) of some of the patterns and elimination (edition) of some others. The decisions about whether re-label or discard depends upon a qualified majority voting among the corresponding $k$-neighborhood (more than $k$ ' out of $k$ ) [9].

On the other hand, pattern re-labeling may introduce new problems because not all discarded prototypes have the same probability of belonging to one of the other classes that may lead to instabilities and unbalanced situations between neighboring classes. To better cope with imperfectly supervised environments, Barandela and Gasca [8] have proposed a combined methodology (Decontamination) in which both class re-labeling and prototype deletion are used in a more intensive way. Decontamination consists of several applications of the Generalized Edition scheme followed by Wilson's editing [3] also reiterated. Decontamination has proved profitable by correcting the training data and cleaning the errors both in the input features and in the class labels [8].

\section{Proposals to Detect Patterns Arising from Missing Classes}

The usual way of dealing with situations in which patterns may exhibit tendencies significantly different from the ones a priori represented in the TS is through outlier detection. In this work, it is intended to go one step further by identifying new classes (or at least new homogeneous groups of patterns). The process will then be divided into two stages. First, in an off-line classification phase, a given TS will be used to classify new patterns but with the ability of adding some of them to the TS. The updated TS (containing one or more newly identified classes) will then be used to classify patterns in the definitive online classification phase. This situation is summarized in Fig. 1.

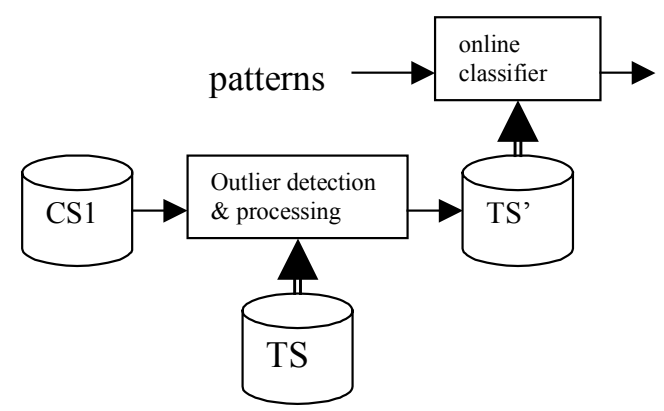

Fig. 1. Schematic representation of the proposed technique. CS1 contains a control set of (unlabelled) patterns possibly containing classes not present in TS 
There are a number of different techniques that are able to detect outliers. Virtually any classifier with a rejection option can be used for this purpose. The main problem is that we have to look for a convenient rejection rate in order to be able to identify new classes into the rejected patterns. Apart from the trivial option of using the $k$-NN rule with a variable rejection rate that is not reported here, a slight modification of the ALIEN technique [2] has been adopted in this study. This method consists of assigning a threshold or weight to each training pattern that relates to the maximum distance at which neighbors can be located with regard to any pattern. That is, given $x$, any neighbor of $x$ farther than its threshold is not considered and will not cast any vote to classify $x$. The procedure consists of two phases, the first one to compute thresholds (learning) and the second one to classify new patterns with reject. The details of both phases are as follows:

Learning phase:

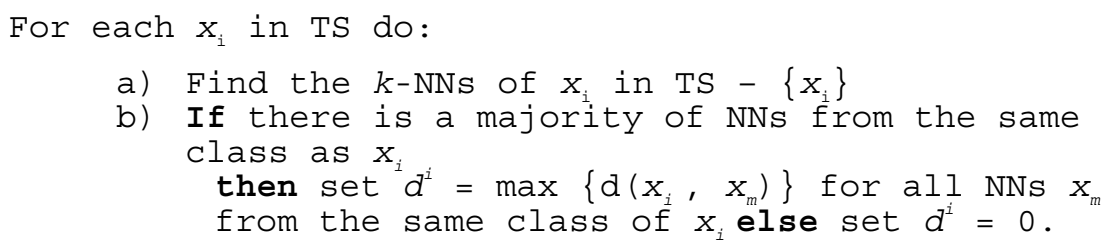

Classification phase:

For every new pattern $X$ to be classified do:

c) Let $X_{u}=\left\{x_{i}\right.$ in $\left.\operatorname{TS} / d\left(X, x_{i}\right) \leq d^{i}\right\}$

d) If $\left|X_{u}\right|=0$, then reject $X$.

else, assign $X$ to the most voted class in $X_{u}$.

The above technique can be seen as an implicit edition of prototypes. A modification of this is proposed in this paper by effectively removing the prototypes on one hand and substituting individual thresholds of patterns by an averaged threshold for the whole class. The resulting technique, which will be referred to as Averaged Distance in this paper, can schematically be explained as follows:

Learning phase:

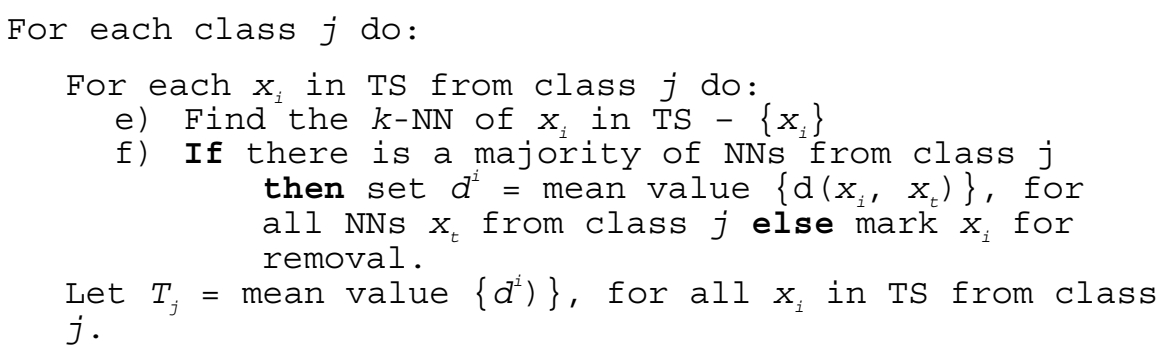

Classification phase (all patterns marked are removed from TS):

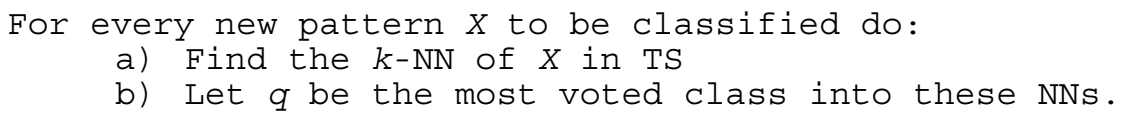




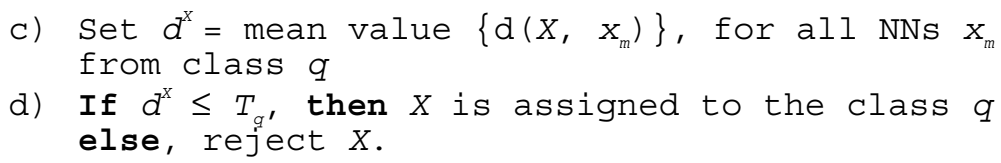

According to our experimentation, this modification of the ALIEN procedure exhibits a more stable behavior and, moreover, can be more efficiently implemented.

\section{Looking for New Classes among Rejected Patterns}

Once a subset of patterns that cannot possibly belong to any of the classes represented in the TS has been identified, we are interested in processing these to see whether some of them are grouped together and can be of interest for future operation of the classifier. In its full generality the problem is very complex but we will make appropriate simplifications in order to carry out this empirical study. First, we will suppose in our experiments that there is always a natural class that has been intentionally omitted in the TS. We will suppose that all classes (including the missing one) have a (normal-like) unimodal distribution.

With these assumptions in mind, the proposed procedure begins the second phase with the application of a clustering algorithm ( $k$-means; [14]). A fixed and reduced number of patterns from each of the classes represented in the TS are joined to the set of the rejected patterns and this augmented set is partitioned into $c+1$ groups (the TS includes $c$ classes). Once the clusters have been identified, we need to match each one of them to the $c$ known classes. In particular, as clusters include some labeled (and representative) prototypes, the majority of these labels are used to assign a particular cluster to a class.

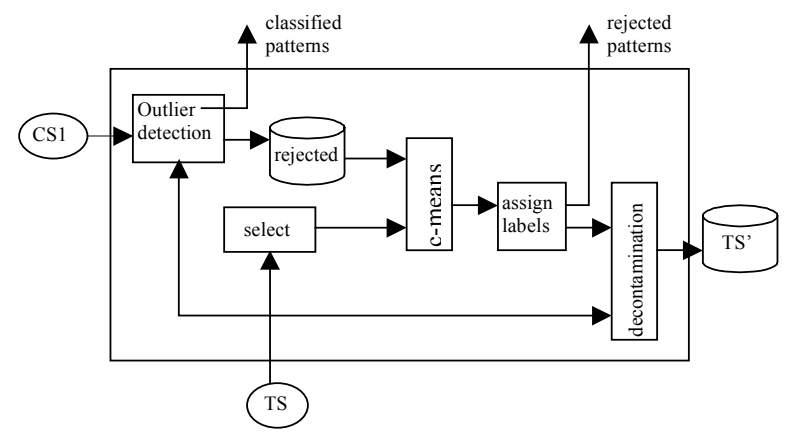

Fig. 2. Schematic description of the proposed method. Given a training set (TS) and patterns to be classified (CS1), the method classifies some of them, rejects other and produces prototypes from a new class and adds them to TS giving rise to TS'

The newly obtained labeled set may contain some patterns close to the boundaries that can increase the classification risk. A further application of the decontamination technique is used then in order to discard some of these prototypes and changing labels of others in order to obtain a "clean" and definitive training set, TS', that can be hopefully used to classify new patters from any of the $c+1$ classes. The whole 
proposed procedure including the first step of detecting potential members of unknown classes is summarized in the block diagram of Figure 2.

It is worth noting that the method splits the input set CS1 into (definitively) rejected patterns, classified patterns and patterns to be added to TS. It is possible as well (as shown in the experiments) to re-classify the rejected patterns using the updated training set TS'. Although this technique has been presented as a procedure taking two input sets and giving one updated TS, the obvious extension is to have a system of this kind operating continuously in an online learning basis.

\section{Experimental Results}

For this empirical study, 24 real datasets taken from the UCI Repository [12] have been considered. In all experiments, one of the classes of each dataset was removed, in turn, to simulate a partially exposed situation.

First, the different techniques to be applied in the first step (outlier detection) were compared. To this end, five replications (different random partitions) were realized for each dataset and for every situation in which one of the classes was eliminated from the training sample. In each of these replications, $80 \%$ of the dataset (but without patterns from one of the classes) was used as training set and the $20 \%$ of the data as the control set. Since the data contained numerical as well as non-numerical features, the Heterogeneous Euclidean Overlap Metric [13] was employed. The parameter value $k=2$ was used for Alien and Averaged Distance. The different databases used are shown in Table 1.

Table 1. Description of the datasets employed in the experiments of phase one. Figures in colums 4 and 9 (TS size) are averaged. Real numbers depend on the class being removed

\begin{tabular}{l|cccc|lcccc}
\hline Dataset & class & feature & TS size & CS size & Dataset & Class & feature & TS size & CS size \\
\hline Iris & 3 & 4 & 80 & 30 & Wine & 3 & 13 & 96 & 34 \\
Glass & 6 & 9 & 150 & 40 & Bridge & 7 & 11 & 78 & 19 \\
Zoo & 7 & 16 & 64 & 15 & Image & 7 & 19 & 314 & 82 \\
Vehicle & 4 & 18 & 504 & 168 & Pima & 2 & 8 & 215 & 153 \\
Cancer & 2 & 9 & 200 & 139 & Liver & 2 & 6 & 160 & 69 \\
Australia & 2 & 14 & 243 & 137 & CRX & 2 & 15 & 246 & 137 \\
Cleveland & 2 & 13 & 110 & 60 & Cardiology & 2 & 9 & 30 & 15 \\
Hungarian & 2 & 13 & 110 & 58 & Beach & 2 & 13 & 75 & 40 \\
Heart & 2 & 13 & 120 & 54 & Swiss & 2 & 13 & 49 & 24 \\
Voting & 2 & 16 & 135 & 86 & Hepatitis & 2 & 19 & 62 & 30 \\
Horse & 2 & 23 & 121 & 59 & Promoter & 2 & 57 & 43 & 20 \\
Ionosphere & 2 & 34 & 141 & 70 & Sonar & 2 & 60 & 78 & 41 \\
\hline
\end{tabular}

The most important characteristic to assess in this part of the work is the capacity of each technique to detect (reject) patterns that do not belong to any of the classes considered in the training sample. These are the results (in percentage) presented in Table 2: mean values (and standard deviation) of each technique for each dataset. Results are analyzed in two aspects: rejected patterns from new classes (those classes not included in the current training sample) and rejected patterns from the known classes (those classes represented in the current training sample). Of course, the 
optimum values would be $100 \%$ of rejected patterns from the new classes and, at the same moment, $0 \%$ of rejected patterns from the known classes.

Unfortunately, these optimum goals are far from being simultaneously reached as results in Table 2 show. The dilemma is that, as the number of patterns from new classes being rejected grows (good sign) it is also increased the number of patterns from known classes remaining unidentified (undesirable effect). In this context, Averaged Distance presents a more convenient tradeoff between these two effects. It is true that this technique also produces a considerable amount of patterns from known classes that remains without classification but this effect is especially good if we aim at extracting information from these rejected patterns. Experiments with other values for the parameter $k$ are not reported here since they did not offer better results. Preprocessing of the training sample through Edition [3] produced some improvements but not in a significant amount.

Table 2. Experimental results -mean values (and standard deviations)- of the percentage of patterns rejected (from the new and the known classes) in each dataset and for each technique assessed

\begin{tabular}{l|cc|cc}
\hline & \multicolumn{3}{|c|}{ New Classes } & \multicolumn{2}{|c}{ Known Classes } \\
\hline Dataset & Alien & Av. Dist. & Alien & Av. Dist. \\
Iris & $82.7(3.7)$ & $98.7(1.8)$ & $15.3(6.4)$ & $34.7(13.0)$ \\
Wine & $76.5(5.5)$ & $100.0(0)$ & $22.7(4.2)$ & $52.7(9.0)$ \\
Glass & $59.0(9.5)$ & $88.5(3.8)$ & $48.1(6.8)$ & $72.4(5.8)$ \\
Bridge & $84.2(9.9)$ & $95.8(4.4)$ & $57.0(12.5)$ & $80.7(9.9)$ \\
Zoo & $94.7(3.0)$ & $100.0(0)$ & $9.6(4.1)$ & $50.4(6.2)$ \\
Image & $90.0(2.5)$ & $99.5(1.1)$ & $22.6(7.6)$ & $44.1(11.7)$ \\
Vehicle & $56.7(1.8)$ & $96.7(0.3)$ & $36.3(3.5)$ & $82.7(2.1)$ \\
Pima & $22.1(5.7)$ & $48.8(2.9)$ & $14.9(2.8)$ & $37.3(2.8)$ \\
Cancer & $52.7(17.4)$ & $85.9(5.0)$ & $11.8(3.3)$ & $35.7(8.7)$ \\
Liver & $21.7(9.0)$ & $43.8(17.5)$ & $18.0(5.8)$ & $39.1(17.4)$ \\
Australian & $32.9(7.2)$ & $89.1(2.1)$ & $17.1(7.1)$ & $49.9(8.1)$ \\
CRX & $28.9(8.0)$ & $89.6(3.0)$ & $20.0(3.9)$ & $51.4(6.5)$ \\
Cleveland & $32.0(9.1)$ & $80.3(6.2)$ & $19.0(9.3)$ & $49.3(6.3)$ \\
Cardiology & $77.3(13)$ & $96.0(4)$ & $24.0(14)$ & $38.7(20)$ \\
Hungarian & $27.6(9.8)$ & $83.5(4.3)$ & $31.4(12.6)$ & $39.3(8.7)$ \\
Beach & $20.5(8.6)$ & $57.5(15)$ & $16.5(8.6)$ & $46.5(16.6)$ \\
Heart & $29.3(3.3)$ & $82.2(8.6)$ & $18.5(5.4)$ & $45.9(5.8)$ \\
Swiss & $25.8(21.9)$ & $62.5(29)$ & $27.5(23.7)$ & $61.7(19.9)$ \\
Voting & $58.8(7.9)$ & $99.5(0.6)$ & $14.0(7)$ & $48.8(11.3)$ \\
Hepatitis & $20.7(9.6)$ & $66.0(15.9)$ & $18.0(6.5)$ & $53.3(11.8)$ \\
Horse & $26.8(5.4)$ & $69.8(3)$ & $19.7(1.9)$ & $54.2(7.3)$ \\
Promoter & $46.0(6.5)$ & $89.0(7.4)$ & $8.0(5.7)$ & $56.0(9.6)$ \\
Ionosphere & $44.6(5.5)$ & $44.6(4.7)$ & $37.8(10.3)$ & $49.7(16.5)$ \\
Sonar & $59.5(5.9)$ & $81.0(8)$ & $19.5(5.7)$ & $46.3(12.2)$ \\
Average & $48.8(2.1)$ & $81.2(2.6)$ & $22.8(1.8)$ & $50.9(1.1)$ \\
\hline
\end{tabular}


Experiments about the whole proposed technique (Figure 2) were carried out using the Averaged Distance method only. Four datasets were selected since it was necessary to employ only those with more than two classes (to correctly simulate a partially exposed environment) and with a sufficient number of patterns in each class. Each dataset was divided into three groups: $50 \%$ for the TS, $25 \%$ for CS1 (the control set to be used in the first step of the procedure) and 25\% for CS2 (control set for second step). Five different random partitions of this kind were performed and their corresponding results averaged in all the experiments. The details of these datasets are shown in Table 3.

Table 3. Description of the real datasets employed in the experiments of Phase two. Figures of TS size are averaged. Real numbers depend on the class being removed

\begin{tabular}{l|ccccc}
\hline Datasets & Classes & Features & TS size & CS1 size & CS2 size \\
\hline Iris & 3 & 4 & 50 & 38 & 37 \\
Wine & 3 & 13 & 59 & 45 & 45 \\
Glass & 6 & 9 & 88 & 54 & 53 \\
Vehicle & 4 & 18 & 316 & 212 & 211 \\
\hline
\end{tabular}

For each partition of the data set into three subsets as explained above, four different methods have been taken into account.

1. The proposed method as shown in Figure 2 and re-classifying the rejected patters from CS1 by using TS'.

2. The same as above but preprocessing the initial TS by using decontamination.

3. External supervision I: which corresponds to using the same $k$-NN rule to classify an independent set corresponding to $50 \%$ of the patterns from known classes and $25 \%$ from the missing one, using a training sample with the same composition.

4. External supervision II: which corresponds to an ideal behavior of the proposed technique. The data sets are split in the same way but the patterns from the missing class in CS1 are identified by hand.

The two last methods are taken into account for comparison purposes. Both methods represents in some way the ideal situation the proposed method is trying to mimic.

Results in Table 4 indicate that the two variants of the proposed procedure excel, in classification accuracy, the behavior of the External Supervision II method. They can also compete in some cases with the External Supervision I, which optimally uses the same amount of information about the missing class available for the proposed method. In fact, in Glass dataset, both variants are more accurate than Ext. Supervision I. In Wine and Vehicle, the variant with initial Decontamination obtains more classification errors than variant 1 . These two datasets present a rather low size/dimensionality ratio that has been reduced even more by the Decontamination methodology. It is important to remark that these variants of the proposed procedure work in a complete automatic way, not requiring the help of a human expert. 
Table 4. Experimental results of the two variants of the proposed procedure. Mean values and standard deviations of the percentage of misclassification

\begin{tabular}{|l|c|c|c||l|l|l|l|}
\hline Iris & \multicolumn{3}{|c|}{ Misclassification } & Vehicle & \multicolumn{3}{|c|}{ Misclassification } \\
\hline Procedure & New & Known & Total & Procedure & New & Known & Total \\
Ext. Sup I & 1.4 & 4.5 & 5.9 & Ext. Sup. I & 6.5 & 13.3 & 19.9 \\
& $(0.3)$ & $(1.3)$ & $(1.6)$ & & $(0.4)$ & $(0.4)$ & $(0.6)$ \\
Ext Sup. II & 17.8 & 3.2 & 21.0 & Ext. Sup. II & 18.1 & 19.6 & 37.6 \\
& $(0.3)$ & $(1.2)$ & $(1.4)$ & & $(0.4)$ & $(1.2)$ & $(0.9)$ \\
Variant 1 & 5.8 & 1.9 & 7.7 & Variant 1 & 12.2 & 13.0 & 25.1 \\
& $(4.6)$ & $(0.6)$ & $(4.7)$ & & $(1.0)$ & $(0.5)$ & $(0.6)$ \\
Variant 2 & 4.4 & 2.1 & 6.6 & Variant 2 & 12.4 & 13.7 & 26.1 \\
& $(4.2)$ & $(1.1)$ & $(3.4)$ & & $(1.7)$ & $(1.2)$ & $(2.0)$ \\
\hline \hline Glass & \multicolumn{2}{|c|}{ Misclassification } & Wine & \multicolumn{2}{|c|}{ Misclassification } \\
\hline Procedure & New & Known & Total & Procedure & New & Known & Total \\
Ext. Sup I & 3.7 & 31.6 & 35.3 & Ext. Sup. I & 1.2 & 3.1 & 4.3 \\
& $(0.5)$ & $(3.0)$ & $(3.0)$ & & $(0.7)$ & $(0.9)$ & $(1.4)$ \\
Ext Sup. II & 11.8 & 27.8 & 39.5 & Ext. Sup. II & 17.6 & 2.3 & 19.9 \\
& $(0.5)$ & $(3.0)$ & $(2.9)$ & & $(0.6)$ & $(0.3)$ & $(0.8)$ \\
Variant 1 & 9.5 & 17.4 & 26.9 & Variant 1 & 7.1 & 2.1 & 9.2 \\
& $(0.6)$ & $(1.6)$ & $(1.6)$ & & $(6.3)$ & $(0.5)$ & $(6.2)$ \\
Variant 2 & 9.4 & 17.3 & 26.7 & Variant 2 & 12.0 & 1.9 & 13.9 \\
& $(0.8)$ & $(1.9)$ & $(1.4)$ & & $(7.4)$ & $(0.8)$ & $(7.2)$ \\
\hline
\end{tabular}

\section{Some Final Comments}

The initial experimental results above reported exhibit the usefulness of the proposed procedure to handle classification situations with incomplete training samples. Working in an automated way, the recognition accuracy of the procedure can compete with the results obtained when help of a human supervisor is available.

The work presented will be extended and improved by retaking some of the decisions and simplifications that have been done here. In particular, the way representative patterns from TS are taken and the identification between classes and clusters when $k$-means is applied have to be revised. Many well studied methods to fully automate our technique and making it more generally applicable exists. Following with this, research is to be done also to adapt our procedure to those cases when two or more classes are not represented in the initial training sample. We intend now to study how to incorporate this idea to an ongoing learning system, for continuously increasing the knowledge of the supervised system, not only with the perception of new classes but also with a better understanding of the already defined classes (see [15]). In these cases, the more difficult problem of distinguishing between pure outliers or noise and rejected patterns that can belong to new classes will arise. 


\section{Acknowledgements}

This work has been partially supported by grants 32016-A from Conacyt and 744.99P from Cosnet, both from Mexico and by the Spanish project TIC2000-1703-C03-03.

\section{References}

1. Dasarathy, B.V.: All you need to know about the neighbors. Proceedings International Conference on Cybernetics and Society, Denver (1979)

2. Dasarathy, B.V.: "There goes the neighborhood": An Alien identification approach to recognition in partially exposed environments. Proceedings of the $5^{\text {th }}$ International Conference on Pattern Recognition (1980)

3. Wilson, D.L.: Asymptotic properties of Nearest Neighbor rules using edited data sets. IEEE Trans. Systems, Man and Cybernetics, SMC-2 (1972) 408-421

4. Barandela, R.: La regla NN con muestras de entrenamiento no balanceadas. Investigación Operacional, X (1) (1990) 45-56

5. Sanchez, J.S., F. Pla and F. Ferri: Prototype selection for the Nearest Neighbor rule through Proximity Graphs. Pattern Recognition Letters, 18, 6 (1997) $507-$ 513

6. Barandela, R.: Una metodología para el reconocimiento de patrones en tareas geólogo-geofísicas. Geofisica Internacional, 34 (4) (1995) 399-405

7. Gasca, E. and R. Barandela: Influencia del preprocesamiento de la muestra de entrenamiento en el poder de generalización del Perceptron Multicapa. 6th Brazilian Symposium on Neural Networks. Río de Janeiro (2000)

8. Barandela, R. and E. Gasca: Decontamination of training samples for supervised pattern recognition methods. In: Advances in Pattern Recognition, Lecture Notes in Computer Science 1876, Springer Verlag (2000) 621-630

9. Koplowitz, J. And T.A. Brown: On the relation of performance to editing in nearest neighbor rules. Proc. 4th Int. Joint Conf. on Pattern Recognition, Japan (1978)

10. Dasarathy, B.V.: Adaptive decision systems with extended learning for deployment in partially exposed environments. Optical Engineering, 34, 5, (1995) 1269-1280

11. Tax, D.M.J. and R.P.W. Duin: Outlier Detection using Classifier Instability. In: A. Amin et al. (eds.), Advances in Pattern Recognition, Lecture Notes in Computer Science, vol 1451, Springer, Berlin (1998)

12. Merz, C.J. and P.M. Murphy: UCI Repository of machine learning databases. University of California at Irvine. http://www.csi.uci.edu/ mlearn (1998)

13. Wilson, D.R. and T.R. Martinez: Reduction Techniques for Instance-Based Learning Algorithms. Machine Learning, 38, 3 (2000) 257-286

14. Duda, R.O. and P.E. Hart: Pattern Classification and Scene Analysis. Wiley, New York (1973)

15. Barandela, R. and M. Juarez: Ongoing learning for Supervised Pattern Recognition. Proceedings of the XIV SIBGRAPI, Brazil, IEEE Press (2001) 5158 